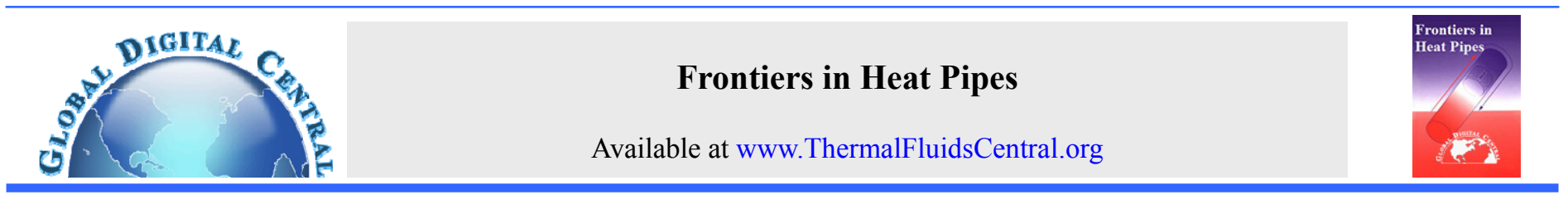

\title{
Miniature High-Temperature Rotating Heat Pipes and Their Applications in Gas Turbine Cooling
}

\author{
Yiding Cao* \\ Department of Mechanical and Materials Engineering, Florida International University, Miami, FL 33174, USA
}

\begin{abstract}
This paper describes an emerging cooling technique of the miniature high-temperature rotating heat pipe (MHTRHP) and its applications in gas turbine engines. The working principle of the MHTRHP and its disposition in the turbine blade and disk are first elaborated, followed by experimental and numerical studies of the subject. The study in the past 10 years indicated that the MHTRHP worked very well under the working temperature environment of a gas turbine blade and disk. Additionally, the MHTRHP is proven to be cost-effective, reliable, and long lasting, which may provide a foundation for the transition from the concept stage to the product development stage of the system.
\end{abstract}

Keywords: Gas Turbine Cooling, Turbine Blade and Disks, Radially Rotating Heat Pipes

\section{INTRODUCTION}

Today, almost all major aircraft, both military and civilian, are powered by gas turbines. Additionally, in 2008 , about $21.3 \%$ of the electricity of the United States was generated by gas turbines burning natural gas. With this large economic scale of the gas turbine industry, a few percentage points of fuel savings could have a significant impact on the fuel demands, pricing, and industry profits. Currently, the gas inlet temperature of almost all advanced gas turbines is well above the maximum allowable metal temperature of the first-stage nozzles and rotor blades. For instance, the maximum allowable metal temperature is on the order of $1200 \mathrm{~K}$, while the current gas inlet temperature is on the order of 1500-1800 K. Under this condition, the first-stage rotor blade must be cooled, and the cooling technology is therefore an enabling technology. In addition to improving energy utilization efficiency, effective cooling could also improve the reliability and integrity of high-speed rotating components. It has been observed that the creep life of turbine blades is reduced to half with every 10 to $15^{\circ} \mathrm{C}$ rise in metal temperature.

Another important issue related to gas turbine blade cooling, particularly in connection with gas turbine power plants, is the utilization of alternative fuels, such as pulverized coal, finely ground biomass, petroleum coke (petcoke), and syngas. Pulverized coal is especially attractive because of its abundant reserves in the United States and its use could reduce the dependence on foreign oils. These lower-grade fuels, however, may contain non-combustible impurities or components that may produce a high percentage of ash in conjunction with pollutants, which may corrode and build deposits on the turbine blades. For these reasons, a viable cooling approach for the rotor blades has yet to be developed.

Like the turbine blades of a gas turbine engine, turbine disks are cooled by compressed air that is bled from the compressor. However, as the turbine is exposed to an increasingly high temperature, the disk rim temperature may also reach a level beyond the maximum allowable temperature. For example, the disk rim temperature of a high-pressure turbine in some development engines has exceeded $1000{ }^{\circ} \mathrm{C}$, which is approaching the creep limitation of the disk material. A literature survey of turbine disk cooling has revealed that although the average air-cooling heat transfer coefficient is generally high, the local heattransfer coefficient at the disk rim is low (Owen, 1988, 1992). A common practice for the cooling enhancement is to utilize a jet impingement at the rim. This approach usually involves the impingement of cooling jets onto the blade attachment region at the rim. Metzger et al. (1979) experimentally studied the jet cooling at the rim of a rotating disk for the enhancement of the heat transfer in that region. The experimental results indicated that the heat transfer rates were unaffected by impingement for small jet flow rates. To gain significant enhancement in heat transfer rates, flow rates of at least onetenth the disk pumping flow capacity is required for a single jet. When a multiple jet array is used, the cooling would consume a relatively large amount of airflow from the compressor. The authors further concluded that many multiple jet rim cooling configurations were probably not very effective in raising the rim cooling rates although they may help to reduce the radial inflow of hot combustion gases. This phenomenon can be explained by the theory of rotationally dominant zones and impingement dominant zones (Metzger and Grochowsky, 1977). When the cooling jet flow rate is low, the jet is apparently swept away by the pumped boundary layer on the disk surface before it has any significant effect on the disk surface. In summary, the jet cooling may be used for the reduction of the maximum temperature at the rim, but it is costly and would consume a relatively large amount of compressed air. It is clear that to significantly reduce the disk rim temperature and achieve a desired turbine engine performance, new cooling approaches should be pursued.

\section{EXISTING COOLING TECHNOLOGIES}


There are a number of available cooling methods that could enable the heat to be removed from a turbine blade. Convection air-cooling and film cooling, in conjunction with certain localized impingement cooling, however, are the primary methods used today in turbine rotor blade cooling. The greatest advantage of the film cooling is its ability to protect the blade surface by a protective layer or film, along its surface, and consequently, the reduction of the heat flux entering the surface. The coolant may be bled at a suitable pressure from the compressor, passed through the internal cooling passages in the blade, and finally discharged from the blade into the mainstream hot gas (Han et al., 2000; Wright et al., 2008; Saha and Acharya, 2007; and Wolf et al., 2001). The coolant flow affects not only the heat transfer, but also the aerodynamic performance of the gas turbine engine. Although the film cooling is a well-established method, difficulty may be encountered for the cooling of the leading or trailing edge of the blade (Bathie, 1984), as well as the handling of the temperature gradient in the stream-wise direction. Another problem associated with the film cooling is the utilization of alternative fuels, particularly in connection with power plant gas turbines. As discussed earlier, alternative fuels such as pulverized coal, finely ground biomass, petroleum coke (petcoke), and syngas are attractive because of their abundant reserves in the United States and renewable sources to reduce the dependence on foreign oils. However, these lower-grade fuels may contain noncombustible impurities or components that may produce a high percentage of ash in conjunction with pollutants, and subsequently corrode and build deposits on the turbine blades. Such deposits can clog the tiny bleed holes for the film cooling and render the film and convective cooling ineffective.

\section{HEAT PIPE COOLING TECHNOLOGY}

A new cooling method that may overcome the difficulties as discussed above is the utilization of rotating high-temperature heat pipes. Gray (1969) is generally credited for the proposal of rotating heat pipes that employ the centrifugal force in the heat pipe to return the condensate in the condenser section to the evaporator section. Since the initial proposal of the rotating heat pipe, extensive studies, both analytical and experimental, have been performed. Notable studies include those of Marto (1976), Maezawa et al. (1981), Daniels and Al-Jumaily (1975), Faghri et al. (1993), and Harley and Faghri (1995). However, most of these studies are related to low-temperature axially rotating heat pipes for the applications of motor shaft cooling and automotive brake system cooling. Gas turbine applications would call for radially rotating heat pipes working at a high temperature and using a liquid metal as the working fluid (Faghri, 1995; Cao, 1996, Faghri et al., 1991a and 1991b; $\mathrm{Cao}$

and

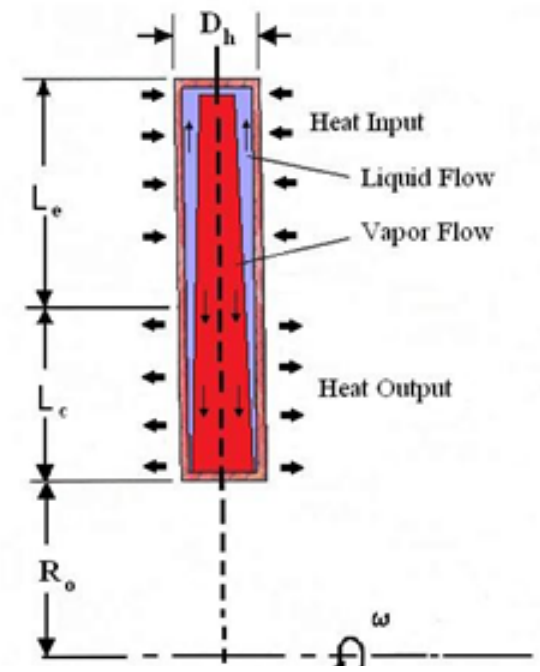

Fig. 1 Schematic of a radially rotating heat pipe.
Faghri, 1993a and 1993b; Cao and Faghri, 1992; Cao and Faghri, 1991; Cao and Chang, 1997; Zuo et al., 1998; Ling and Cao, 2001; Ling and Cao, 2000; and Cao et al., 2009).

A miniature rotating heat pipe (Cao, 1996), as shown in Fig. 1, is generally disposed in an orientation perpendicular to a shaft and rotates
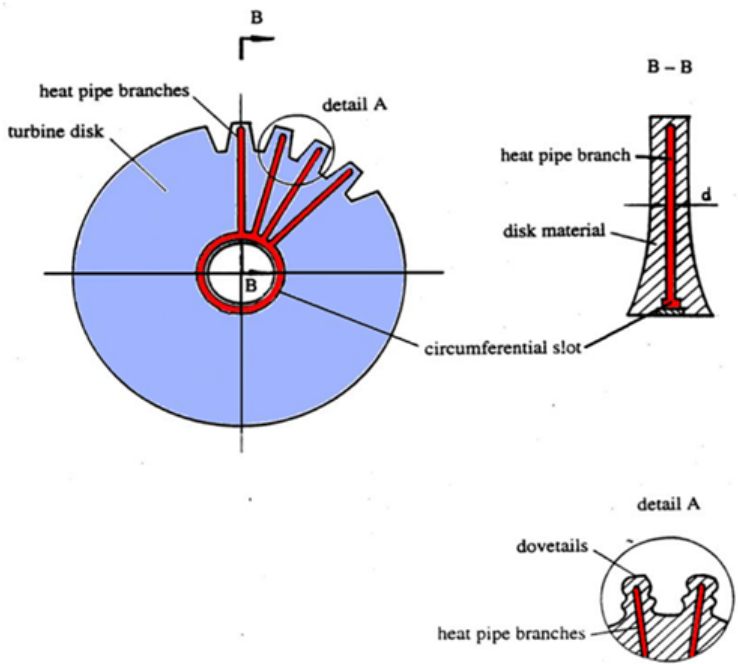

Fig. 2 Schematic of a gas turbine disk with embedded rotating heat pipes.

along with the shaft, in which the upper section serves as an evaporator $\left(L_{e}\right)$, while the lower section $\left(L_{c}\right)$ serves as a condenser. The condensate in the condenser is pumped back to the evaporator by centrifugal force. The greatest advantages of this type of heat pipe are its simple structure and high heat transfer capacity. The heat pipe in this case is nothing but an empty cavity, without a wick structure, which is filled with a small amount of the working fluid. The shell of the cavity is also the same material as the turbine blade or disk being cooled. Due to the high rotating speed of the turbine shaft, the centrifugal force generated may be several orders of magnitude greater than that of the gravitational force. As a result, the heat transfer capacity and reliability may be a few orders of magnitude greater than that of a conventional heat pipe employing gravity or capillary force as its driving force. In the following, some typical applications of the radially rotating heat pipe in gas turbines are illustrated.

Figure 2 schematically illustrates a turbine disk with a number of radially rotating heat pipes embedded in the disk (Cao, 1997). The heat pipes are circumferentially arranged and extend radially from the disk rim towards the inner radius of the disk. The diameter of the heat pipe may be less than $3 \mathrm{~mm}$ on the basis of disk strength considerations. For a gas turbine employing the traditional dovetail attachment, the maximum disk temperature usually occurs near the tip of the dovetail. As a result, the heat pipe is extended as close to the dovetail tip as possible provided that strength considerations are satisfied. A heat pipe arrangement of the type under consideration is elaborated in detail A of Fig. 2. The spaces between the individual heat pipes could be reserved, among other considerations, for rotor blade cooling air passages. To facilitate the processing and filling of the heat pipes, the disk incorporates a circumferential slot that works as a reservoir for the working fluid. As indicated in the figure, the circumferential slot or reservoir is located near the inner radius of the disk. Through this reservoir, the individual heat pipe branches are interconnected, and these heat pipes essentially become the branches of the heat pipe system in the disk. As a result of this interconnection, the whole disk becomes a single heat pipe system that can be processed and filled with working fluid only once, and the fabrication cost of the disk can be substantially reduced.

Figure 3 demonstrates an example of combined film cooling/heat pipe cooling for gas turbine rotor blades (Cao, 1996) (air bleed at the leading edge is not shown). Notice that a miniature radially rotating 

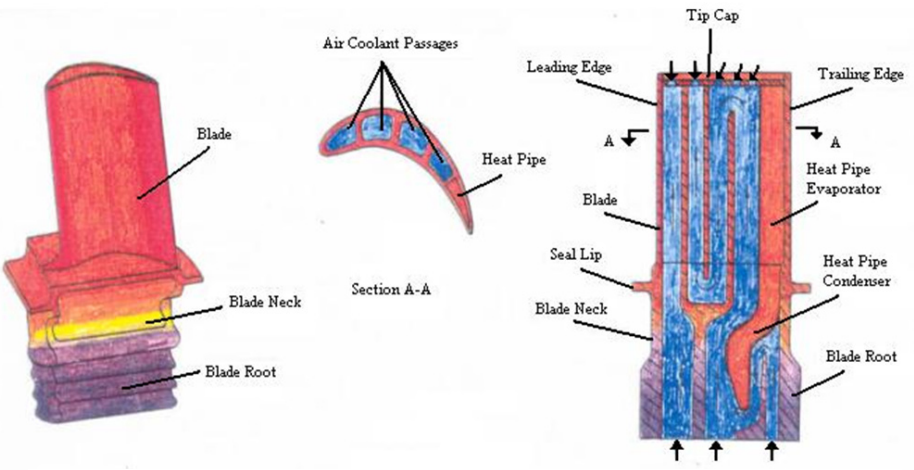

Fig. 3 Combined air/heat pipe cooling for a gas turbine rotor blade.

heat pipe is incorporated at the trailing edge of the rotor blade in conjunction with the traditional air cooling method in an effort to enhance the temperature reduction at the trailing edge. The integration of the heat pipe in the trailing edge also reduces the cooling air bleed from the trailing edge, and therefore, more cooling air may be supplied to the leading edge of the blade to reduce the high temperature there. Similarly, miniature heat pipes may be incorporated at the leading edge or other locations of the turbine blade for the purpose of the temperature reduction as well as temperature uniformity, as shown in Fig. 4. In practical applications, all these heat pipes may be interconnected through a liquid reservoir near the tip to facilitate the heat pipe processing and working fluid filling.

In 1996, Cao (1996) initiated the concept of miniature hightemperature radially rotating heat pipes for gas turbine blade cooling and since then has conducted a series of experimental and analytical studies (Cao and Faghri, 1994; Cao, 1996; Ling and Cao, 2001; Ling and Cao, 2000; and Cao et al., 2009). However, before the rotating heat pipe is employed for gas turbine cooling the performance of the heat pipe must be fully validated in terms of heat transfer characteristics and reliability over a long period of time for practical applications. For these purposes, two tubular miniature high-temperature rotating heat pipes were designed, fabricated, and experimentally studied along with

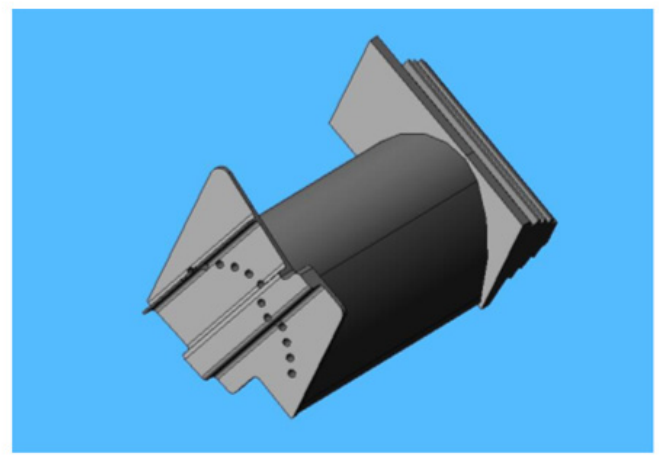

Fig. 4 A Turbine blade showing the locations of miniature heat pipes.

corresponding analytical and numerical studies (Ling and Cao, 2001; Ling and Cao, 2000; and Cao et al., 2009). The objectives of studying the tubular heat pipes, like that shown in Fig. 1, are to identify the performance characteristics of the heat pipe under high temperature and high-speed rotating conditions and validate the reliability of the heat pipe as well as the compatibility of the working fluid with the heat pipe shell. The tubular heat pipe, filled with sodium, has an inner diameter on the order of $1 \mathrm{~mm}$, and is suitable to be embedded in a turbine rotor blade or disk. The configuration and dimensions of the heat pipe are shown in Fig. 5. The attachment on the left with screws is for assembling the heat pipe to the rotor of the rotating rig. The first tubular heat pipe has an inner diameter of $2.0 \mathrm{~mm}$ and is filled with about 0.070 grams of sodium (labeled as heat pipe No. 1). The second heat pipe has an inner diameter of $1.5 \mathrm{~mm}$ and is filled with almost the same amount of sodium (labeled as heat pipe No. 2).

Figure 6 illustrates the rotating rig used in the experimental study. In the experimental study, the major parameters that could be adjusted are the revolutions per minute of the test apparatus, heat pipe operating temperatures, heat inputs to the evaporator section, and cooling-air flow rate in the condenser section. The vibration of the apparatus was also controlled to ensure a safe operation. As illustrated in Fig. 6, the shaft is driven by a motor [1] having a maximum rotating speed of 3,600 rpm. In order to dampen the vibration of the rotor, a flexible coupling [18] is used to connect the shaft to the motor. The springs [3] connecting the rotor and frame have a significant damping function when the rotor

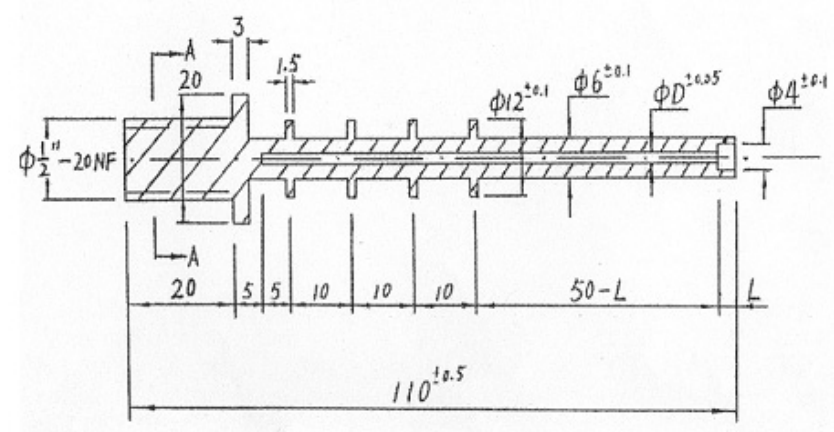

Fig. 5 Configuration of a tubular miniature heat pipe studied.

system experiences slight imbalances, which would cause vibration otherwise. As a result, the rotating test apparatus constructed is of certain self-balance capability. Two slip-ring assemblies [7] are mounted on the shaft. One is used to supply the electrical current from the power transformer to the heater [15] that provides the heat input to the heat pipe evaporator, and the other is used to feed the temperature data from the thermocouples mounted on the heat pipe shell to the data acquisition system. The rotor disk of the apparatus consists of an inner cylinder [10] and an outer cylinder [11]. The inner cylinder is mounted on the shaft, and the outer cylinder is in turn fixed onto the inner
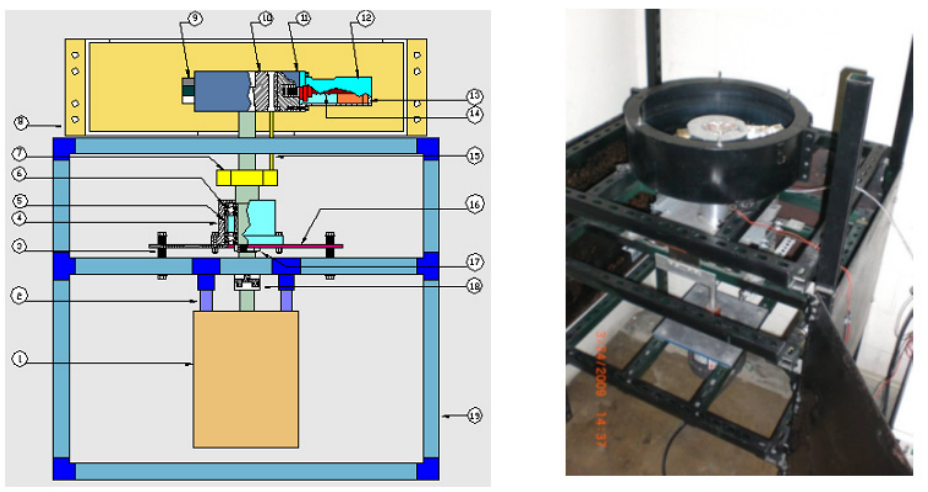

Fig. 6 Schematic (left) and a photo (right) of the rig used in the tests.

cylinder. The miniature radially rotating high-temperature heat pipe [14] is mounted in the outer cylinder. To balance the rotor system, a counter weight [9] is also mounted in the cylinder opposite the heat pipe. The heater cap [12] connected to the outer cylinder has two functions: one is for the protection of the heater, and the other is for adjusting the cooling-air flow rate over the condenser section. This is accomplished through the adjustment of the cooling-air flow window 
on the heater cap. If the diameter of the outer cylinder is extended, the rotating radius of the heat pipe can be increased.

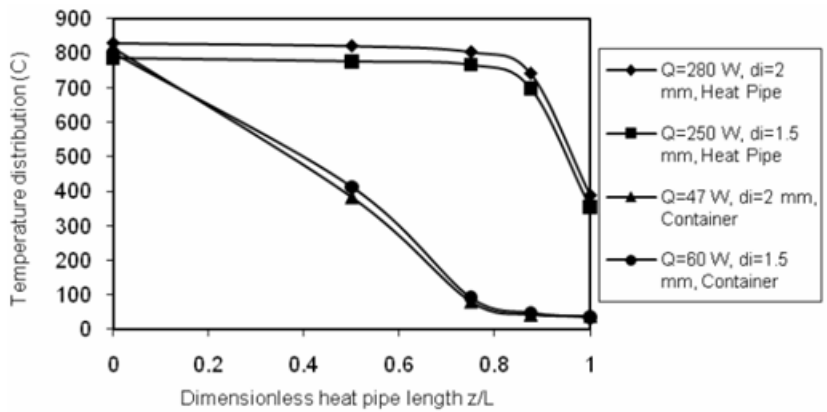

Fig. 7 Typical testing results of 1999 for the two heat pipes as compared to the results of the empty heat pipe container.

The two heat pipes were tested between 1998-1999 and some typical results with a heat input of up to $280 \mathrm{~W}$ are illustrated in Fig. 7. Because of the small diameter of the vapor space, the heat flux at the liquid-vapor interface reached nearly $100 \mathrm{~W} / \mathrm{cm}^{2}$, which is typical of a gas turbine cooling condition. Some testing results of the heat pipe container at a heat input of $60 \mathrm{~W}$, which represent pure conduction without heat pipe functionality, are also included in the figure. As can be seen from the figure, the heat pipes functioned very well, except for the end of the condenser section. It is believed that the sudden drop in temperature at the end of the heat pipe condenser is due to the larger attachment to the rotor of the rotating rig as shown on the left of Fig. 5, which acts as a large heat sink. The effect of the attachment can also be seen from the temperature of the heat pipe container. On the other hand, some non-condensable gas may exist in the heat pipe, which may also have caused this sudden drop. The same two heat pipes were re-tested in 2007, and some associated results during the heat pipe startup processes are shown in Figs. 8 and 9. A thermal resistance analysis indicated that the thermal resistance of the heat pipe was only $1 / 17$ that of the heat pipe shell (container) material. In other words, the heat transfer capacity of the heat pipe can be increased by more than 17 times. Due to the fact that when the inner diameter of a heat pipe approaches zero, the heat pipe performance would approach that of the heat pipe shell, the comparison between the heat pipe and the heat pipe shell could be much more favorable if the inner diameter of the heat pipe is larger. Because of the small inner diameter, which is on the order of 1.5-2.0 mm, the volume occupied by the heat pipe vapor space, which represents the penalty of using a heat pipe in a turbine blade or disk, is less than $1 / 10$ of the total volume of the heat pipe. This indicates that significant heat transfer improvement using a heat pipe is achieved with a very small volume penalty. On the other hand, if only the inner diameter of the heat pipe is used for the basis of comparison, the thermal resistance of the heat pipe could be hundreds of times lower

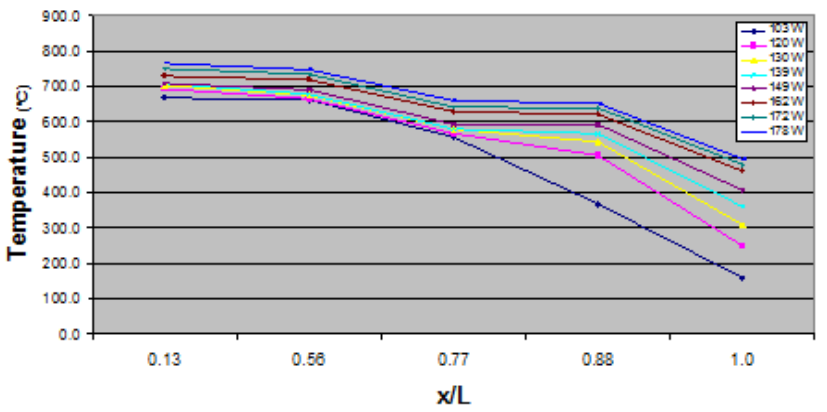

Fig. 8 Typical test results of 2007 for heat pipe No. 1 (test run \# 1).

than that of the shell or blade /disk material. Additionally, the tests in
2007 revealed that the performance of the heat pipes matched that in 1998, and no degradation of the heat pipes performance were detected over a time period of 9 years, which validated the expectation of the heat pipes' reliability due to its simple and wickless structure. The

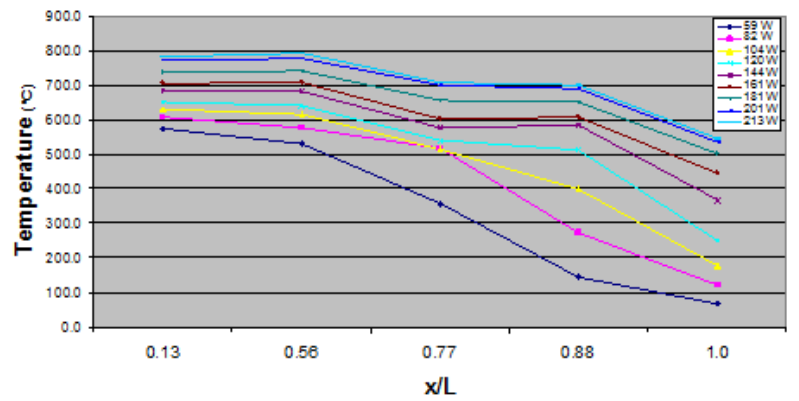

Fig. 9 Typical test results of 2007 for heat pipe No. 1 (test run \# 2).

safety of the sodium heat pipe is also well documented. In a gas turbine cooling application, even if there is a leakage in the heat pipe, the sodium would be burned off harmlessly in the hot gas without causing any problems because of the very small amount of sodium that needs to be filled inside the heat pipe.

Numerical studies have also been performed to simulate the performance of a gas turbine disk incorporating a number of heat pipes (Cao and Ling, 2000) as shown in Fig. 10. In addition, the numerical results were compared with those of a conventional disk without incorporating the heat pipes as shown in Fig. 11. The results indicate that a temperature reduction of $100-250{ }^{\circ} \mathrm{C}$ as compared to those

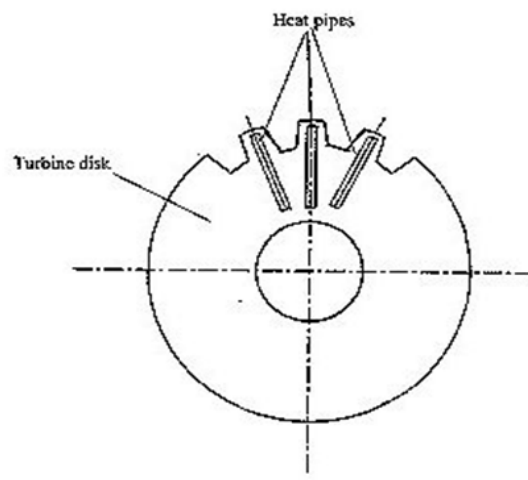

Fig. 10 Schematic of a gas turbine disk incorporating rotating heat pipes.

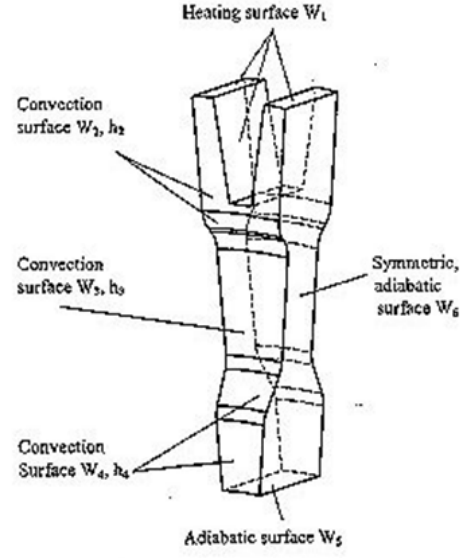

(a) The turoire disk wift the dovetait.

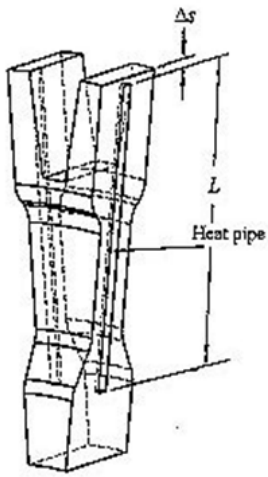

(b) The turbine disk jacorporating radially rotating heat pipes.
Fig. 11 Schematic of turbine disks with and without incorporating heat pipes. 
without the heat pipes can be achieved under different disk cooling conditions.

\section{DISCUSSIONS AND CONCLUSIONS}

- $\quad$ Test results of both 1998 and 2007 as well as the numerical simulations indicate that the miniature rotating hightemperature heat pipes work very well under the working temperature environment of a gas turbine blade and disk.

- It is believed that the results are significant in terms of providing a new cooling method for gas turbine engines to reduce fuel consumption and make a contribution to $\mathrm{CO}_{2}$ emission reduction.

- For a heat pipe to be introduced in a product, it must be cost effective, reliable, and long lasting. This is the case for lowtemperature water heat pipes in connection with electronics cooling. Today, tens of millions of heat pipes are being used in laptop and desktop computers every year, which is believed to be one of the most significant achievements by the heat transfer community in the past 30 year.

- Due to its simple structure, the miniature high-temperature rotating heat pipe can be economically fabricated within turbine blades and disks. The heat pipe is wickless and high centrifugal pumping forces are available for condensate return. Therefore the heat pipe would work reliably and would not encounter any working limitations. The heat pipe is also long lasting as evidenced by the testing results of 1998 and 2007 as presented in this paper. No apparent performance degradation after nearly 10 years!

- More research is needed in terms of fundamental studies and prototype developments to refine this new cooling technique and make a transition from this conceptual stage to product development stage.

\section{REFERENCES}

Bathie, W.W., Fundamentals of Gas Turbines, John Wiley and Sons, New York, 1984.

Cao, Y., 1996, "Rotating Micro/Miniature Heat Pipes for Turbine Blade Cooling Applications," AFOSR Contractor and Grantee Meeting on Turbulence and Internal Flows, September 1996, Atlanta, GA.

Cao, Y., 1997, "A Feasibility Study of Turbine Disk Cooling by Employing Radially Rotating Heat Pipes," Final Report for Summer Faculty Research Program (Wright Laboratory),sponsored by Air Force Office of Scientific Research and Turbine Engine Division, Wright Laboratory.

Cao, Y. and Faghri, A., 1991, "Transient Multidimensional Analysis of Nonconventional Heat Pipes with Uniform and Nonuniform Heat Distributions," ASME J. Heat Transfer, Vol. 113, pp. 995-1002. doi:10.1115/1.2911233

Cao, Y. and Faghri, A., 1992, "Closed-Form Analytical Solutions of High- Temperature Heat Pipe Startup and Frozen Startup Limitation," ASME J. Heat Transfer, Vol. 114, pp. 1028-1035.

doi: $10.1115 / 1.2911873$
Cao, Y. and Faghri, A., 1993a, "A Numerical Analysis of HighTemperature Heat Pipe Startup from the Frozen State," ASME J. Heat Transfer, Vol. 115, pp. 247-254.

doi:10.1115/1.2910657

Cao, Y. and Faghri, A., 1993b, "Conjugate Modeling of High Temperature Nosecap and Wing Leading Edge Heat Pipes," ASME J. Heat Transfer, Vol. 115, pp. 819-822.

doi:10.1115/1.2910765

Cao, Y. and Faghri, A., 1994, "Micro/Miniature Heat Pipes and Operating Limitations," Journal of Enhanced Heat Transfer, Vol. 1, No. 3, pp. 265-274.

Cao, Y. and Chang, W.S., 1997, "Analyses of Heat Transfer Limitations of Radially Rotating Heat Pipes for Turbomachinery Applications," AIAA 32nd Thermophysics Conference, AIAA 97 - 2542, June 23-25, Atlanta, GA.

Cao, Y. and Ling, J., 2008, “An Experimental Study of Micro Radially Rotating Heat Pipes with Water as the Working Fluid," Proceedings of ASME MNHT2008, Micro/Nano Scale International Heat Transfer Conference, January 6-9, 2008, Tainan, MNHT2008 -52115.

Cao, Y., Ling, J., Rivir, R., and MacArthur, C., 2000, "A Numerical Analysis of Gas Turbine Disks Incorporating Rotating Heat Pipes," Proceedings of ASME International Mechanical Engineering Congress and Exposition, Vol. 3, pp. 61-67, Orlando, Florida.

Cao, Y., Gao. M., and Reding, B., 2009, "Experimental Studies of Rotating Heat Pipes for Cooling Gas Turbine Rotors and Disks," AIAA paper No. 2009 - 1427.

Daniels, T. and Al-Jumaily, F., 1975, "Investigations of the Factors Affecting the Performance of a Rotating Heat Pipe," Int. J. Heat Mass Transfer, Vol. 18, pp. 961-973. doi:10.1016/0017-9310(75)90190-8

Faghri, A., 1995, Heat Pipe Science and Technology, Taylor and Francis, Washington D.C.

Faghri, A., Buchko, M., and Cao, Y., 1991a, "A Study of High Temperature Heat Pipes with Multiple Heat Sources and Sinks, Part I: Experimental Methodology and Frozen Startup Profiles," ASME J. Heat Transfer, Vol. 113, pp. 1003-1009.

doi:10.1115/1.2911193

Faghri, A., Buchko, M., and Cao, Y., 1991b, "A Study of High Temperature Heat Pipes with Multiple Heat Sources and Sinks, Part II: Analysis of Continuum Transient and Steady State Experimental Data with Numerical Predictions," ASME J. Heat Transfer, Vol. 113, pp. 1010-1016

doi:10.1115/1.2911194

Faghri, A., Gogineni, S., Thomas, S., 1993, "Numerical Analysis of Vapor Flow in an Axially Rotating Heat Pipe," American Society of Mechanical Engineers, Vol. 221, pp. 11-21.

Gray, V.H., 1969, "The Rotating Heat Pipe - A Wickless, Hollow Shaft for Transferring Heat Fluxes," Proc. ASME/AIChE Heat Transfer Conf., Minneapolis, pp. 1-5, ASME Paper No. 69-HT-19.

Harley, C. and Faghri, A., 1995, "Two-Dimensional Rotating Heat Pipe Analysis,” ASME J. Heat Transfer, Vol. 117, No. 1, pp. 202-208. doi: $10.1115 / 1.2822304$ 
Han, J.C., Dutta, S., and Ekkad, S.V., 2000, Gas Turbine Heat Transfer and Cooling Technology, Taylor and Francis, New York.

Harley, C. and Faghri, A., 1995, "Two-Dimensional Rotating Heat Pipe Analysis,” ASME J. Heat Transfer, Vol., 117, No. 1.

Incropera, F.P., Dewitt, D.P., Bergman, T.L., and Lavine, A.S., 2007, Introduction to Heat transfer, Jone Wiles \& Sons, New York.

Ling, J. and Cao, Y., 2001, "Experimental Investigations of a Radially Rotating Miniature High-Temperature Heat Pipe for Turbine Blade Cooling," ASME Journal of Heat Transfer, Vol. 113, pp. 113-119.

doi:10.1115/1.1332777

Ling, J. and Cao, Y., 2000, "Closed-Form Analytical Solutions for the Radially Rotating Miniature High-Temperature Heat Pipes including Non-Condensable Gas Effects," Int. J. Heat Mass Transfer, Vol. 43, No. 19, pp. 3661-3671.

doi:10.1016/S0017-9310(99)00339-7

Ling, J., Cao, Y., and Chang, W.S., 1999, "Analysis of Radically Rotating High-Temperature Heat Pipes for Turbomachinery Application," ASME Journal of Engineering for Gas Turbines and Power, Vol. 121, pp. 306-312.

doi:10.1115/1.2817121

Ling, J., Cao,Y., Rivir, R. and MacArthur, C., 2002, "Analytical Investigations of Rotating Disks with and without Incorporating Rotating Heat Pipes," ASME Journal of Engineering for Gas Turbines and Power, Vol. 126, pp. 680-683.

doi: $10.1115 / 1.1760528$

Maezawa, S. Suzuki, Y., and Tsuchida, A., 1981, "Heat Transfer Chracterisitics of Disk-Shaped Rotating Wickless Heat Pipes," Proc. 4th International Heat Pipe Conferences, London, pp. 725-733.
Marto, P., 1976, "Performance Characteristics of Rotating Wickless Heat Pipes," Proc. 2nd Int. Heat Pipe Conf., Bologna, pp. 281-291.

Metzger, D.E., Mathis, W.J., and Grochowsky, L.D., 1979, "Jet Cooling at the Rim of a Rotating Disk," Journal of Engineering for Power, Vol. 101, pp. 68-72.

Metzger, D.E. and Grochowsky, L.D., 1977, "Heat Transfer Between an Impinging Jet and a Rotating Disk," ASME Journal of Heat Transfer, Vol. 99, No. 4.

Owen, J.M., 1988, “Air-Cooled Gas-Turbine Discs: a Review of Recent Research,” Int. J. Heat and Fluid Flow, Vol. 9, No. 4, pp. 354-365. doi:10.1016/0142-727X(88)90001-X

Owen, J.M., 1992, "Recent Developments in Rotating-Disc Systems," ImechE, pp. 83-92.

Saha, A.K. and Acharya, S., 2007, "Turbulent Heat Transfer in Ribbed Coolant Passages of Different Aspect Ratios: Parametric Effects," ASME Journal of Heat Transfer, Vol. 129, pp. $449-463$. doi: $10.1115 / 1.2709653$

Wolf, W.B. de, Woldendorp, S., and Tinga, T., 2001, "Analysis of Combined Convective and Film Cooling on an Existing Turbine Blade," National Aerospace Laboratory NLR, NLR-TP-2001-148.

Wright, L.M., Liu, Y.H., Han, J.C., and Chopra, S., 2008, "Heat Transfer in Trailing Edge, Wedge-Shaped Cooling Channels under High Rotating Numbers," ASME Journal of Heat Transfer, Vol. 130, 0717011.

doi: $10.1115 / 1.2907437$

Zuo, Z.J., Faghri, A., and Langston, L., 1998,"Numerical Analysis of Heat Pipe Turbine Vane Cooling," ASME Journal of Engineering for Gas Turbines \& Power, Vol. 120, pp. 735-743. 